\title{
Cardiovascular risk and metabolic profile of Polish citizens from Lower Silesia. First signs of metabolic crisis?
}

Lukasz Kozera ${ }^{1,2}$, Wiktor Kuliczkowski ${ }^{2,3}$, Elzbieta Gocek ${ }^{1,2}$

\author{
${ }^{1}$ Łukasiewicz Research Network - PORT Polish Center for Technology Development, \\ Wroclaw, Poland \\ 2BBMRI.pl \\ ${ }^{3}$ Department and Clinic of Cardiology, Wroclaw Medical University, Wroclaw, Poland
}

Submitted: 1 April 2019; Accepted: 14 August 2019

Online publication: 14 October 2020

Arch Med Sci 2022; 18 (3): 617-623

DOI: https://doi.org/10.5114/aoms.2020.99922

Copyright $\odot 2020$ Termedia \& Banach

\begin{abstract}
Introduction: Population biobanks are essential for the development of public health screening and improvement of personalized medicine. Since 2012, Biobank of Łukasiewicz Research Network - PORT Polish Center for Technology Development (PORT Biobank) has collected more than 120000 biological samples from nearly 5000 inhabitants of Lower Silesia, together with a variety of demographic, anthropometric, life style and health information.

Material and methods: The analyzed group consisted of 2274 participants (1398 women, 876 men). Both women and men were further subdivided into five age decades $(20+, 30+, 40+, 50+, 60+)$. For this study, the level of lipids (total cholesterol, high-density lipoprotein (HDL), low-density lipoprotein (LDL), triglycerides) was estimated and correlated with the level of high-sensitivity C-reactive protein (hs-CRP) and biometric parameters.

Results: We have demonstrated for the first time that biochemical changes that may lead to cardiovascular diseases (CVD) occurred already in the group of people aged 30+. Our observation is based on measurements of lipids, glucose, inflammatory (hs-CRP) and biometric markers such as body mass index (BMI) and waist-to-hip ratio (WHR).

Conclusions: Positive correlations with age for these variables suggest the ongoing progress of metabolic changes, which in the end may lead to a fatal outcome such as myocardial infarction or stroke. It suggests that CVD screening programs should be dedicated to a wider group, especially younger citizens, in order to prevent fatal outcomes related to CVD.
\end{abstract}

Key words: cardiovascular diseases, metabolic crisis, population-based screening, biobanking.

\section{Introduction}

Population-based biobanks are essential for the development and monitoring of public health [1-5]. Poland became a member of the biggest biobanking infrastructure in Europe (BBMRI-ERIC) in 2016 and a large proportion of public funds has been dedicated towards development of biobanking infrastructures [6]. Indeed there is an ongoing effort towards building regional biobanks and longitudinal cohorts which can be used for the development of precision medicine and profiling of disease screening programs $[7,8]$. The population-based study conducted on citizens of Lower Silesia (2012-2016) is one of the first projects performed by the newly established PORT Biobank, created due to lack

\author{
Corresponding author: \\ Elzbieta Gocek \\ Łukasiewicz Research \\ Network - PORT Polish \\ Center for Technology \\ Development \\ Wroclaw, Poland \\ E-mail: \\ elzbieta.gocek@port. \\ lukasiewicz.gov.pl
}


of population-based cohorts in this area. The research profile is defined by high incidence of cardiovascular diseases (CVD) already observed in other Polish studies (WOBASZ I, WOBASZ II and NATPOL - National Multicenter Health Survey and Arterial Hypertension and other CVD risk factors in Poland, TERCET, REGARDS) [9-13]. The PORT Biobank population consisted initially of almost 4000 participants aged $20-70$ years with a main scope to characterize the risk of CVD and diabetes on the basis of biomarker and biometric measurements. The additional aspect that convinced us to create such a program was related to the fact that the younger generation (younger than 40 years old) is not eligible for some screening programs, despite the fact that during the last few decades eating habits as well as level of physical activity have markedly changed. As described previously [14], a reduced fat and cholesterol diet was reported only by every $5^{\text {th }}$ patient and a low calorie diet by every $100^{\text {th }}$ participant in the WOBASZ study. Furthermore, the quality of nutrition and health knowledge in subjects with diagnosed CVDs in the Polish population turned out to be insufficient. Another study also reported that intake of saturated fat, sugar and complex carbohydrates is a cause for concern especially in Eastern European countries [15]. Impaired glucose (GLUC) level among European societies has a direct effect on total CVD incidence, and together with an elevated level of low-density lipoprotein cholesterol (LDL-C) potentially accelerates atherosclerosis $[13,16,17]$. Moreover, physical activity including active commuting (i.e. walking, cycling) of Polish citizens remains unsatisfactory, despite promotion of a healthy lifestyle [18]. For example about $66-68 \%$ of Finnish or Danish citizens report practicing vigorous sport once a week in contrast to $28 \%$ of Poles [19]. Taking into consideration risk factors mentioned above, we may assume that intervention actions such as broad screening for CVD and diabetes is required and should also be

Table I. Sex distribution and quantitative parameters of the studied cohort. The study group consisted of 2274 participants divided into five age decades $(20+, 30+, 40+, 50+, 60+)$. Distributions of sex and quantitative information in relation to decades are shown in the table

\begin{tabular}{|lcc|}
\hline Age decade & $\begin{array}{c}\text { Women } \\
(n=1398)\end{array}$ & $\begin{array}{c}\text { Men } \\
(n=876)\end{array}$ \\
\hline $20+$ & $460(68.15 \%)$ & $215(31.85 \%)$ \\
\hline $30+$ & $261(54.26 \%)$ & $220(45.74 \%)$ \\
\hline $40+$ & $274(58.55 \%)$ & $194(41.45 \%)$ \\
\hline $50+$ & $263(64.30 \%)$ & $146(35.70 \%)$ \\
\hline $60+$ & $140(58.09 \%)$ & $101(41.91 \%)$ \\
\hline
\end{tabular}

addressed to the younger generation. Monitoring of diet and physical activity, as well as regular measurement of blood pressure, lipids and other CVD markers, should be performed even at the age of thirty, in order to determine the critical time point when the human body stops compensating side effects of the modern life style [20, 21]. Attenuated atherosclerosis is strongly related to inflammatory status. Hence the relationship between level of lipids and high-sensitivity C-reactive protein (hs-CRP) has been associated with adverse cardiovascular events due to vascular inflammation and formation of atherosclerotic plaque [22, 23].

The aim of the study was to characterize metabolic changes and determine the age when the first symptoms of metabolic crisis occur in the population of inhabitants from the Lower Silesia region. Our population-based study employs a novel approach such as a broad spectrum of metabolic data and incorporation of the youngest participants from the age of twenty and is one of the first to be organized in Poland.

\section{Material and methods}

\section{Study group}

The initial cohort of volunteers who decided to participate in the PORT Biobank study consisted of 4098 individuals, aged 20-70, which is about $1 \%$ of the local population. All participants who declared any chronic disease (for example high blood pressure, CVD, diabetes, thyroid problems), regular use of medicines or those individuals who had an elevated level of CRP (> $10 \mathrm{mg} / \mathrm{dl}$ ) were excluded from the data analysis. We did not exclude patients on the basis of smoking status or physical activity as the idea of the study was to present a typical cross-sectional population. The final group consisted of 2274 participants (1398 women, 876 men). Both women and men were further subdivided into five age decades $(20+, 30+, 40+$, $50+, 60+$ ). Detailed distribution of sex in relation to decades of age and quantitative information about the cohort are shown in Table I. The study protocol was examined and consent to proceed was granted by the supervising Bioethical Committee (decision numbers: 73/2012, 482/2015, 477/2016 and 607/2017). Informed consent was obtained from all participants prior to sample collection.

\section{Sample collection}

Fasting blood samples were collected using the BD Vacutainer collection system (plastic tubes with spray-coated silica for serum extraction) according to the manufacturer's protocol. Serum for biobanking and routine in vitro diagnostic (IVD) laboratory testing was spun at $2500 \mathrm{~g}$ for $15 \mathrm{~min}$ at 
room temperature. Serum isolation was performed at a maximum time of $4 \mathrm{~h}$ after blood collection.

\section{Body metrics}

Body metrics and a medical questionnaire (including familial history of CVD and diabetes) were recorded for each study participant. Weight and height were used to calculate body mass index (BMI) according to the following formula: $\mathrm{BMI}=$ weight $[\mathrm{kg}]$ divided by the square of height [m] [24]. Waist and hip measurements [cm] were used to calculate waist-to-hip ratio (WTH).

\section{Lipid profile}

Level of total cholesterol (tCHOL), triglycerides (TG) and high-density lipoprotein cholesterol (HDL-C) were determined by enzymatic assay using colorimetric methods performed on the fully automated biochemical analyzer Cobas Integra 400 plus System from Roche Diagnostics GmbH. All measurement steps were performed according to the manufacturer's protocols (cholesterol $2^{\text {nd }}$ generation test ID 0-586; triglycerides test ID 0-010; HDL $3^{\text {rd }}$ generation, test ID 0-331). Low-density lipoprotein cholesterol level was calculated with the Friedewald formula: $L D L-C[\mathrm{mmol} / \mathrm{l}]=$ total cholesterol $[\mathrm{mmol} / \mathrm{l}]$ - (HDL-C $[\mathrm{mmol} / \mathrm{l}]+$ triglycerides $[\mathrm{mmol} / \mathrm{l}] / 2.2)$, when TG level is lower than $4.5 \mathrm{mmol} / \mathrm{l}$.

\section{High-sensitivity C-reactive protein level}

Level of hs-CRP was measured by the high-sensitivity method on the fully automated biochemical analyzer Cobas Integra ${ }^{\circledR} 400$ plus System from Roche Diagnostics $\mathrm{GmbH}$. All measurement steps were performed according to the manufacturer's protocols (CRPHS, test ID 0-033). In brief, the method was based on particle-enhanced (latex) immunological agglutination and turbidimetric precipitate determination.

\section{Glucose}

Level of GLUC was measured photometrically on the Cobas c System from Roche Diagnostics $\mathrm{GmbH}$. The enzymatic reference method with hexokinase was performed according to the manufacturer's protocol (Glucose HK $3^{\text {rd }}$ generation, test ID 056831 6).

\section{Statistical analysis}

The $R$ package for Windows (version 3.5) was used for statistical calculations [25]. The ShapiroWilk test and Q-Q plot were used to assess normality of data. Due to an unacceptable deviation from normality we used nonparametric tests in our study. In order to analyze obtained data, the Kruskal-Wallis test was performed. To verify differences between age groups, pairwise comparison using the post-hoc Nemenyi test with $\chi^{2}$ approximation for independent samples was adopted. Spearman's rank correlation between age groups and variation level was estimated. A $p$-value of $\leq 0.05$ was acknowledged as significant.

\section{Results}

In order to evaluate the prevalence of metabolic crisis and its components (such as lipids and GLUC impaired level, abdominal obesity or hs-CRP expression level) as CVD risk factors in the Lower Silesia population, we collected and examined serum samples for lipid and GLUC profile, as well as hs-CRP level. For each donor, biometric parameters were also measured. The group characteristics of PORT Biobank participants are shown in Table I. It is important to underline that we excluded from the statistical analysis those who might suffer from or who reported any chronic disease that leads to increased CVD risk. Certainly there is a higher number of women amongst all participants (61.48\% vs. $38.52 \%)$, who are more willing to participate in the study (Table I).

Lipid profile in different groups of donors showed major changes that are correlated with age (Figure 1). Total cholesterol, TG and LDL-C concentration increased along with maturity. Taking into account upper reference limits for these metabolites (URL) recommended by ESC/EAS guidelines [26], donors from the 30+ group already reached values higher than the URL for total cholesterol. Moreover, total cholesterol level exceeded reference values with an increasing tendency regarding age (Figure 1). An increasing concentration of TG was noted in all studied age groups, but it did not exceed the URL in any of them.

LDL-C level, the major driver for the development of atherosclerotic plaque, demonstrated higher than URL values in the group of donors aged $40+$, with an increasing tendency correlated with age. Level of HDL-C did not decrease below reference values in any age group studied (Figure 1).

The next step was to evaluate changes in body metrics that may be correlated with lipid level and may lead to the observed increasing obesity in the Lower Silesia population and increased risk of CVD. Taking into account the URL for this measurement we observed elevated BMI already in the $30+$ group and an increasing tendency correlated with age (Figure 2). A similar tendency was observed in the WHR measurements (exceeding reference values evaluated for women from the groups aged 30+ up to 60+). Additionally, hs-CRP and GLUC levels were measured, and an increasing tendency regarding all age groups was noted, and all values could indicate increased risk for future CVDs (Figure 2). 


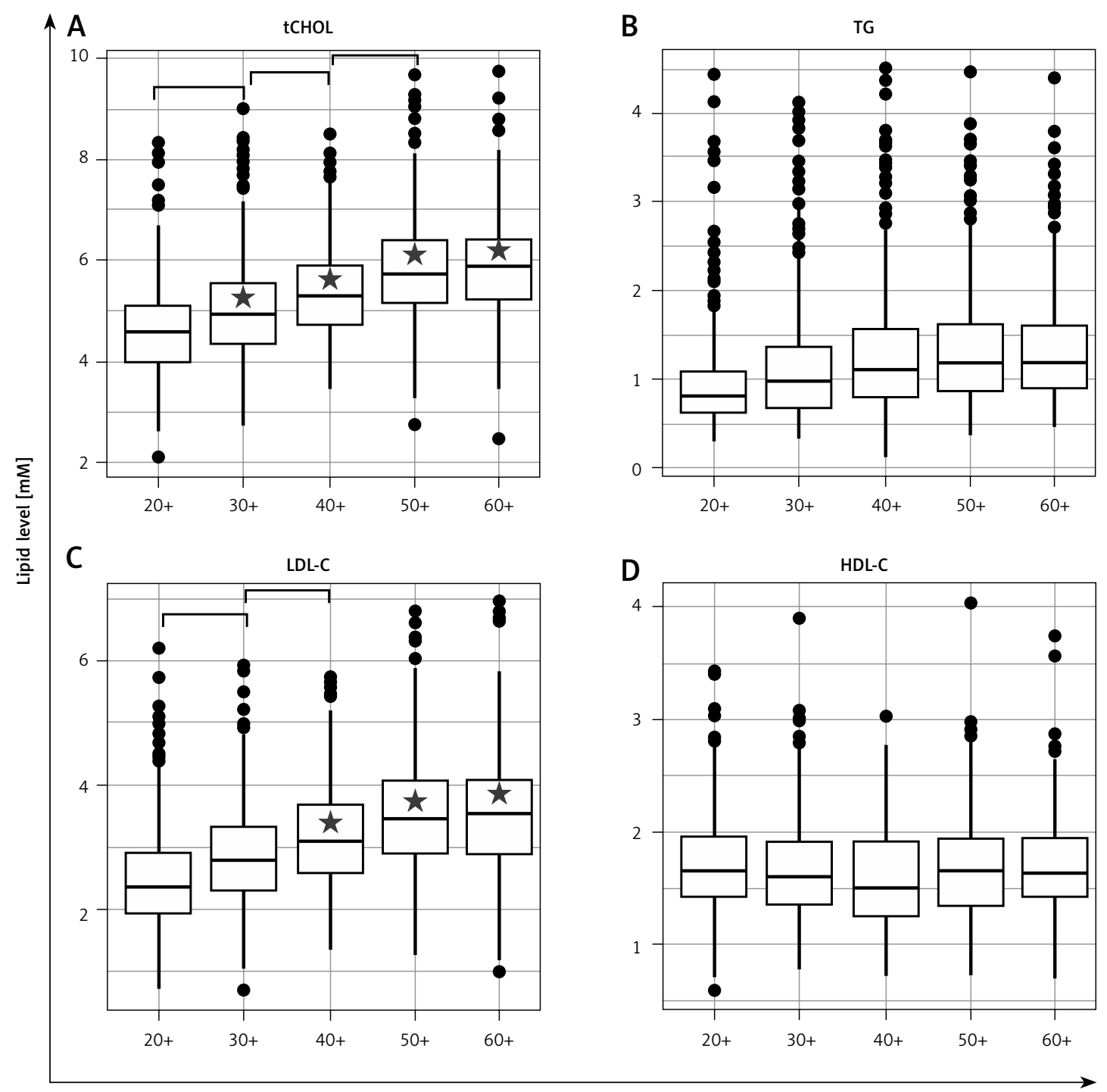

Age group [decade]

Figure 1. Changes in lipid profile relative to age groups in Lower Silesia inhabitants. Serum lipid profile, $\mathbf{A}-$ total cholesterol (tCHOL), B - triglycerides (TG) and D - high-density lipoprotein cholesterol (HDL-C) levels were measured by enzymatic assay, as described in Material and methods section. C - Low-density lipoprotein cholesterol (LDL-C) level was calculated with Friedewald formula. Results exceeding reference values are indicated with a star. Values that increased significantly between particular age decades are indicated with a linker (Pearson's $\chi^{2}$ test; $p<0.0001)$. Reference values for: tCHOL 2.97-4.91 mM, TG 0.56-1.69 mM, LDL-C $<2.97 \mathrm{mM}, \mathrm{HDL}-\mathrm{C}>1.03 \mathrm{mM}$

To further confirm obtained results indicating increased risk of CVD in Lower Silesia subjects, correlation analysis between age groups and lipids, hs-CRP, GLUC levels and biometric parameters was performed (Table II). A noteworthy correlation was observed between age groups and tCHOL, TG, LDL-C, hs-CRP, GLUC, BMI, and WHR, demonstrating that the level of metabolites responsible for CVD development changes and is correlated with alteration in body composition.

\section{Discussion}

The leading cause of mortality in the $21^{\text {st }}$ century is coronary heart disease $[27,28]$. More importantly, premature ischemic heart disease is an increasing cause of hospitalization among populations living in developed countries, including Poland $[29,30]$. The socio-economic impact triggered by both hospitalization and recovery after myocardial infarction is vast, especially among patients during their period of professional activity [31, 32]. The problem we face today is the lack of efficient screening programs, capturing individuals with the initial stages of CVD, as well as inefficient monitoring of success rate related to the change of life style and sometimes pharmacotherapy. Studying the literature related to the development of cardiovascular biomarkers in relation to the prediction of clinical end-points and the final outcome, still lipids, hs-CRP, GLUC, body metric 

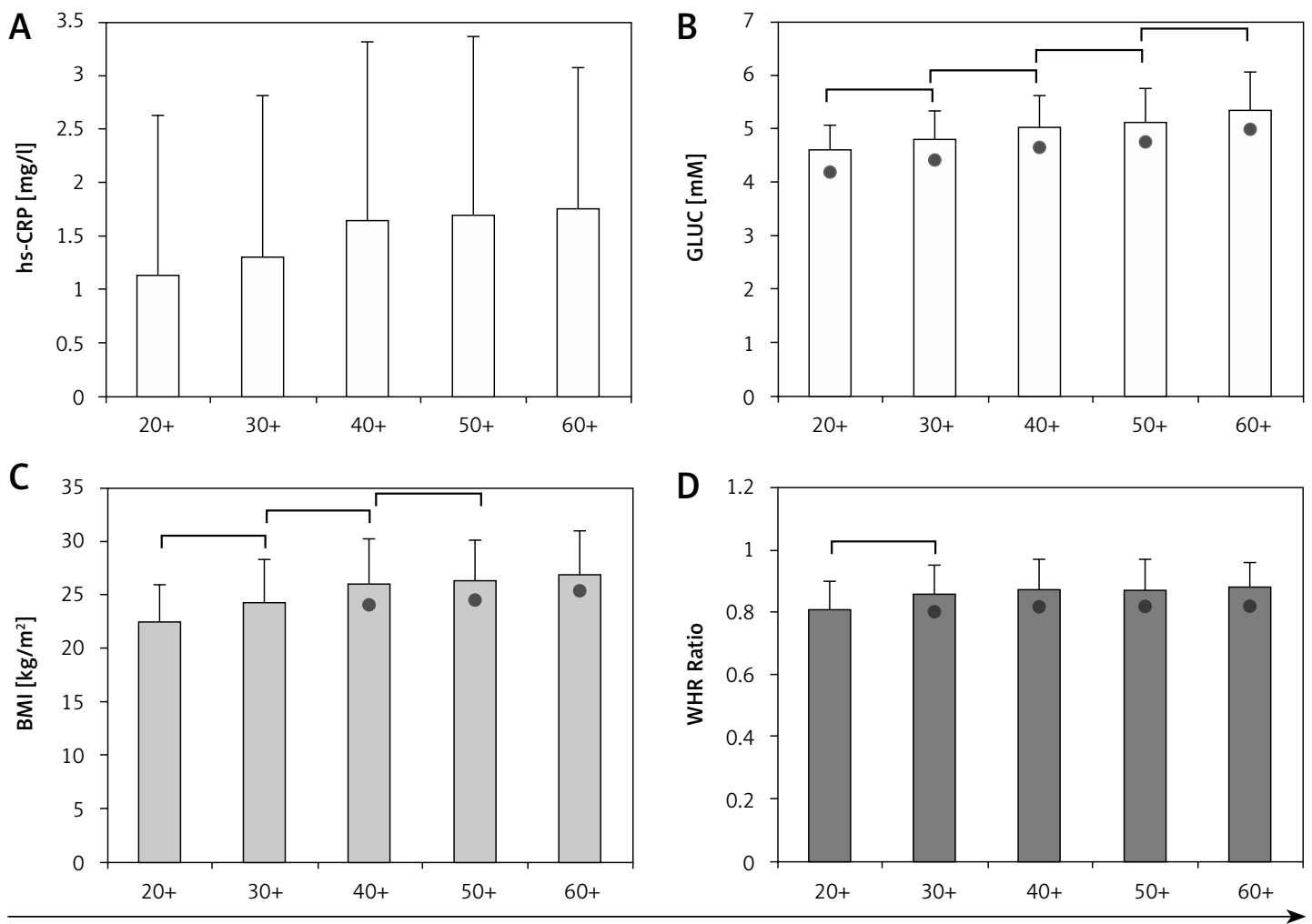

Age group [decade]

Figure 2. A - High-sensitivity C-reactive protein (hs-CRP), B - glucose (GLUC), C - body mass index (BMI) and D - waist-to-hip ratio (WHR) profiles in relation to age decades. Serum hs-CRP level was measured by immunological agglutination and turbidimetric precipitate determination; GLUC level was measured by enzymatic reaction with hexokinase; BMI and WHR were calculated as described in Material and methods section. Results exceeding reference values are indicated with a dot. Values that increased significantly between particular age decades are indicated with a linker (Pearson's $\chi^{2}$ test; $p<0.0001$ ). Reference values for: hs-CRP 0-3.0 mg/l, GLUC: 3.6-5.8 mM, BMI 18.5-24.99, WHR for men $\leq 0.09$; WHR for women $\leq 0.085$

measurements, blood pressure, life style and familial history remain crucial [33-35]. That brings us to the conclusion that appropriate tools which are relatively low-cost are available. In fact, it is important to create an entire algorithm and early detection national program that could guarantee a better outcome seen as a reduction of CVD cases including myocardial infarction, especially in the younger age group.

Our data showed that inhabitants of Lower Silesia who were included in the study group (about $1 \%$ of the local population) are willing to participate in preventive programs, and a greater proportion are women than men, which probably results from various psychological barriers. For the first time we documented the so-called metabolic crisis seen in study participants aged 30+ onward when compared to younger individuals. Previous studies on CVD incidence in Poland did not include such a young population and division of the cohort into such narrow age groups. Our observation was based on measurements of lipids, GLUC, inflammatory and biometric markers such as hs-CRP, BMI and WHR. The tendency to exceed reference values of $\mathrm{tCHOL}$ seen in the $30+$ group
Table II. Correlation between age and lipid level, hs-CRP and biometric parameters. Spearman's rank correlation between age groups $(n=2274)$ together with correlation coefficient $(\rho)$ was calculated. $P$-values $<0.0001$ are indicated in bold

\begin{tabular}{|lcc|}
\hline Parameter & \multicolumn{2}{c|}{ Age groups $(n=2274)$} \\
\cline { 2 - 3 } & $P$-value & $\rho$ \\
\hline tCHOL & $<0.0001$ & 0.471 \\
\hline TG & $<0.0001$ & 0.318 \\
\hline LDL-C & $<0.0001$ & 0.45 \\
\hline HDL-C & 0.05 & -0.0414 \\
\hline hS-CRP & $<0.0001$ & 0.277 \\
\hline BMI & $<0.0001$ & 0.428 \\
\hline WHR & $<0.0001$ & 0.280 \\
\hline
\end{tabular}

tCHOL - total cholesterol, TG - triglycerides, LDL-C - low-density lipoprotein cholesterol, HDL-C - high-density lipoprotein cholesterol, hs-CRP - high-sensitivity C-reactive protein, BMI - body mass index, WHR - waist-to-hip ratio.

and LDL-C seen in the 40+ group correlated well with changes in the body composition and obesity observed in these groups. These may be very valu- 
able data that address important issues and may have an impact on the clinical practice in Poland. Increasing BMI, WHR and the level of TG (although still within the URL) affect the liver function and attenuate lipogenesis, leading to the overload of adipocytes. That is the first stage of metabolic syndrome, which accelerates other signaling cascades. Consequently we observed an increase in the hs-CRP level with age, since the fat tissue acts as an endocrine organ promoting synthesis of inflammatory cytokines such as IL-6, which mediates synthesis of CRP in the liver [36]. Dysfunction of lipid homeostasis together with expression of inflammatory molecules is closely linked to severe metabolic disorders, metabolic syndrome and CVD, such as myocardial infarction or stroke [37]. Observations performed on different populations showed that this is a mechanism that cannot be ceased completely, but should be identified at the earliest stage, as it can be reduced by making lifestyle changes by increasing physical activity and changing dietary habits. [19]. Our data in some aspects are very consistent with other studies performed in Poland such as WOBASZ I, WOBASZ II and NATPOL, but we characterized each age group more deeply to accurately determine the age decade at which the metabolic crisis may occur $[9,38]$. In fact until now there is not a prevention program to control CVD risk factors among the younger generation, such as our study group. The idea of screening programs often fails to keep up with dynamic environmental changes, and too little attention is paid to those groups of patients who could actually benefit from them. Biobanks due to their broad approach to population-based research may influence the public health policy and may improve early CVD diagnosis and effective prevention.

The study set up by PORT Biobank continues. The current research protocol for the cohort also involves monitoring of blood pressure and measurement of insulin resistance (using the HOMA calculation) that begin to confirm our initial findings based on body metric measurements and an increasing level of TG (data not shown). The analyzed metabolic profile indicated that more specific testing (i.e. ultrasound imaging) could be performed during current recruitment such as carotid-artery intima media thickness. We are considering monitoring of dietary habits and physical activity in a longitudinal project, which may perhaps answer more precisely the question when the metabolic crisis occurs and will clearly indicate the age of metabolic crisis shift towards the younger generation in the future. CVDs must be targeted at a primary health promotion level and traditional and relatively inexpensive tools play a substantial role in population-based health monitoring.

\section{Acknowledgments}

This research was funded by the Ministry of Science and Higher Education of Poland, grant number DIR/WK/2017/2018/01-1. We thank Professor Leszek Kalinowski for valuable comments and critical review of the manuscript.

\section{Conflict of interest}

The authors declare no conflict of interest.

\section{References}

1. Harris JR, Burton P, Knoppers BM, et al. Toward a roadmap in global biobanking for health. Eur J Hum Genet 2012; 20: 1105-11.

2. Vartiainen E. The North Karelia Project: cardiovascular disease prevention in Finland. Glob Cardiol Sci Pract 2018; 2018: 13.

3. Sigurdardottir FD, Lyngbakken MN, Holmen OL, et al. Relative prognostic value of cardiac troponin I and C-reactive protein in the general population (from the Nord-Trondelag Health [HUNT] Study). Am J Cardiol 2018; 121: 949-55.

4. Bycroft C, Freeman C, Petkova D, et al. The UK Biobank resource with deep phenotyping and genomic data. Nature 2018; 562: 203-9.

5. Berkelmans GFN, Gudbjörnsdottir S, Visseren FLJ, et al. Prediction of individual life-years gained without cardiovascular events from lipid, blood pressure, glucose, and aspirin treatment based on data of more than 500000 patients with type 2 diabetes mellitus. Eur Heart J 2019; 40: 2899-906.

6. Witon M, Strapagiel D, Glenska-Olender J, et al. Organization of BBMRI.pl: The Polish Biobanking Network. Biopreserv Biobank 2017; 15: 264-9.

7. Holub P, Swertz M, Reihs R, van Enckevort D, Muller H, Litton JE. BBMRI-ERIC Directory: 515 biobanks with over 60 million biological samples. Biopreserv Biobank 2016; 14: 559-62.

8. Paskal W, Paskal AM, Debski T, Gryziak M, Jaworowski J. Aspects of modern biobank activity - comprehensive review. Pathol Oncol Res 2018; 24: 771-85.

9. Zdrojewski T, Solnica B, Cybulska B, et al. Prevalence of lipid abnormalities in Poland. The NATPOL 2011 survey. Kardiol Pol 2016; 74: 213-23.

10. Drygas W, Niklas AA, Piwonska A, et al. Multi-centre National Population Health Examination Survey (WOBASZ II study): assumptions, methods, and implementation. Kardiol Pol 2016; 74: 681-90.

11. Piwonska A, Piotrowski W, Kozela M, et al. Cardiovascular diseases prevention in Poland: results of WOBASZ and WOBASZ II studies. Kardiol Pol 2018; 76: 1534-41.

12. Dyrbus K, Gasior M, Desperak P, Nowak J, Osadnik T, Banach M. Characteristics of lipid profile and effectiveness of management of dyslipidaemia in patients with acute coronary syndromes - data from the TERCET registry with 19,287 patients. Pharmacol Res 2019; 139: 460-6.

13. Penson P, Long DL, Howard G, et al. Associations between cardiovascular disease, cancer, and very low high-density lipoprotein cholesterol in the REasons for Geographical and Racial Differences in Stroke (REGARDS) study. Cardiovasc Res 2019; 115: 204-12.

14. Waskiewicz A, Szczesniewska D, Szostak-Wegierek D, et al. Are dietary habits of the Polish population consistent 
with the recommendations for prevention of cardiovascular disease? - WOBASZ II project. Kardiol Pol 2016; 74: 969-77.

15. Boylan S, Welch A, Pikhart H, et al. Dietary habits in three Central and Eastern European countries: the HAPIEE study. BMC Public Health 2009; 9: 439.

16. Stratton IM, Adler Al, Neil HA, et al. Association of gly caemia with macrovascular and microvascular complications of type 2 diabetes (UKPDS 35): prospective observational study. BMJ 2000; 321: 405-12.

17. Uusitupa M, Peltonen M, Lindstrom J, et al. Ten-year mortality and cardiovascular morbidity in the Finnish Diabetes Prevention Study - secondary analysis of the randomized trial. PLoS One 2009; 4: e5656.

18. Kwasniewska M, Pikala M, Bielecki W, et al. Ten-year changes in the prevalence and socio-demographic determinants of physical activity among Polish adults aged 20 to 74 years. Results of the National Multicenter Health Surveys WOBASZ (2003-2005) and WOBASZ II (2013-2014). PLoS One 2016; 11: e0156766.

19. Special Eurobarometer 412 Report; European Comission: 2014.

20. Dai S, Huang B, Zou Y, Liu Y. Associations of dipping and non-dipping hypertension with cardiovascular diseases in patients with dyslipidemia. Arch Med Sci 2019; 15 $337-42$.

21. Gonzalez FEM, Ponce-Rulz N, Rojas-Garcla AE, et al. PON1 concentration and high-density lipoprotein characteristics as cardiovascular biomarkers. Arch Med Sci Atheroscler Dis 2019; 4: e47-e54.

22. Whelton SP, Roy P, Astor BC, et al. Elevated highsensitivity C-reactive protein as a risk marker of the attenuated relationship between serum cholesterol and cardiovascular events at older age. The ARIC Study. Am J Epidemiol 2013; 178: 1076-84.

23. Penson PE, Long DL, Howard G, et al. Associations between very low concentrations of low density lipoprotein cholesterol, high sensitivity C-reactive protein, and health outcomes in the Reasons for Geographical and Racial Differences in Stroke (REGARDS) study. Eur Heart J 2018; 39: 3641-53.

24. Eveleth PB. Physical status: the use and interpretation of anthropometry. Report of a WHO Expert Committee WHO. Am J Hum Biol 1996; 8: 786-7.

25. R Core Team. R: A language and environment for statistical computing. R Foundation for Statistical Computing, Vienna, Austria. 2018.

26. Catapano AL, Graham I, De Backer G, et al. 2016 ESC/ EAS guidelines for the management of dyslipidaemias. Rev Esp Cardiol (Engl Ed) 2017; 70: 115.

27. World Health Organisation Report. https://www.who. int/en/news-room/fact-sheets/detail/the-top-10-causes-of-death.

28. Roth GA, Johnson C, Abajobir A, et al. Global, regional, and national burden of cardiovascular diseases for 10 causes, 1990 to 2015. J Am Coll Cardiol 2017; 70: 1-25.

29. Levit RD, Reynolds HR, Hochman JS. Cardiovascular disease in young women: a population at risk. Cardiol Rev 2011; 19: 60-5.

30. Maroszynska-Dmoch EM, Wozakowska-Kaplon B. Clinical and angiographic characteristics of coronary artery disease in young adults: a single centre study. Kardiol Pol 2016; 74: 314-21.

31. Calvo M, Subirats L, Ceccaroni L, Maroto JM, de Pablo C, Miralles F. Automatic assessment of socioeconomic impact on cardiac rehabilitation. Int J Environ Res Public Health 2013; 10: 5266-83.
32. Schroder SL, Fink A, Richter M. Socioeconomic differences in experiences with treatment of coronary heart disease: a qualitative study from the perspective of elderly patients. BMJ Open 2018; 8: e024151.

33. Yusuf S, Hawken S, Ounpuu S, et al. Effect of potentially modifiable risk factors associated with myocardial infarction in 52 countries (the INTERHEART study): case-control study. Lancet 2004; 364: 937-52.

34. Winter MP, Blessberger $\mathrm{H}$, Alimohammadi $\mathrm{A}$, et al. Long-term outcome and risk assessment in premature acute myocardial infarction: a 10-year follow-up study. Int J Cardiol 2017; 240: 37-42.

35. Soran H, Adam S, Mohammad JB, et al. Hypercholesterolaemia - practical information for non-specialists. Arch Med Sci 2018; 14: 1-21.

36. Vegiopoulos A, Rohm M, Herzig S. Adipose tissue: between the extremes. EMBO J 2017; 36: 1999-2017.

37. Banach M, Jankowski P, Jozwiak J, et al. PoLA/CFPiP/PCS guidelines for the management of dyslipidaemias for family physicians 2016. Arch Med Sci 2017; 13: 1-45.

38. Pajak A, Szafraniec K, Polak M, et al. Changes in the prevalence, treatment, and control of hypercholesterolemia and other dyslipidemias over 10 years in Poland: the WOBASZ study. Pol Arch Med Wewn 2016; 126: 642-52. 